\title{
Application and Optimization of MIMO Communication in Wide Area Monitoring Systems
}

\author{
Youcef Grainat ${ }^{\mathrm{a}, 1, *}$, Abdelmadjid Recioui ${ }^{\mathrm{a}, 2}$ \\ ${ }^{a}$ Laboratory of Signals and Systems (LSS), Institute of Electrical and Electronic Engineering, University M'hamed Bougara of Boumerdes, Avenue de \\ l'indépendance, 35000, Boumerdes, Algeria. \\ 1y.grainat@univ-boumerdes.dz ${ }^{2}$ a_recioui@univ-boumerdes.dz \\ * corresponding author
}

\section{ARTICLE INFO}

Article history

Received May 8, 2020

Revised June 7, 2020

Accepted June 10, 2020

Keywords

smart grid communication

MIMO

optimization

whale optimization algorithm

\section{ABSTRACT}

Multiple-Input Multiple-Output (MIMO) technology uses a multitude of antennas at both transmitter and receiver to transfer a larger data mount simultaneously. It is the key technology in the 4th and 5th communication system generations. In this work, the use of MIMO technology to enhance the data transfer in terms of completeness, correctness and latency in Wide Area Monitoring Systems (WAMS) is envisaged. To further enhance the system, optimization is done to design the communication system in terms of physical layout. A comparison with the state of art technologies is done to highlight how the adoption of the MIMO technology would enhance the data transfer within the smart grid.

This is an open access article under the CC-BY-SA license.

\section{Introduction}

The advanced information and communication technologies (ICTs) tend to become extensively embedded to our daily life. One key area witnessing on this is the proliferating bright age of electricity termed the intelligent grid or more known as the Smart Grid (SG). Compared to the actual grid, the Smart Grid incorporates a bi-directional flow of information, automation and control. It is characterized by optimized energy efficiency through the reciprocal real-time exchange of information between utility company and the users based on the deployment of advanced sensors, smart meters and the information technology (IT) [1-6].

The smart grid can be regarded as a layered user-involved platform, comprising the power system layer, a control section, a communication link, a security section and an application layer. The communication link ensures a two-way communication in the smart grid system. It is one of the most crucial agents that enable smart grid applications. The smart grid can be cast as an involved collection of networks, involving both high voltage and communication infrastructures along with various intelligent electronic devices (IEDs) [7-8]. Communication networks constitute the required infrastructure enabling a utility to monitor and control these devices from a remote location. In the smart grid system, diverse communication technologies and architectures are brought together. Communication networks should satisfy some desired properties such as reliability, latency, bandwidth and security, corresponding to each smart grid application. The involvedness of the smart grid may result in complications in selecting right communication networks because of the large number of parameters and diverse requirements to be taken into account in relation to the applications and utility expectations. 
Wide-area monitoring, wide-area control and wide-area protection utilize Wide Area Networks and turn out to be the next-generation key to improve power system planning, operation and protection in the smart grid. These applications employ the system broad data and wisely chosen local information to oppose the spread of harming disturbances [1]. Wide-area monitoring, control and protection applications present higher data resolution and shorter response time than the classical supervisory control and data acquisition (SCADA) and energy management (EMS) systems. Wide-area monitoring, control and protection applications provide high-resolution data contrary to SCADA/EMS which offers a measurement update interval of several seconds (or even minutes).

The IEEE Standard for Synchrophasors for Power Systems (IEEE Std.C37.118 provides definitions of measurement and data transmission formats for real-time data reporting in electric power systems [9]. For wide-area monitoring applications, the size containing measurements made by a PMU has a minimum message of 52 bytes. The required response time for wide area monitoring applications is in the range of milliseconds to minutes, and the requirement on communication system reliability is very high.

Though wide-area protection and control applications present more advanced protection/control systems in comparison to the traditional power systems, more rigorous performance and availability requirements are needed. As an illustration, the required response time for widearea protection and control applications should be in the range of milliseconds to minutes, and the communication system reliability requirement should be very high. The typical message size can vary according to the employed communication protocols. Examples of protocols used for these applications include MIRRORED BITS, IEC 61850, Generic Object-Oriented Substation Event (GOOSE), and ETHER-CAT communications [10].

Due to the importance of the communication infrastructure in the success of smart grid implementation and operation, there has been a variety of research works in the literature focusing on this topic. The authors of $[1,11-13]$ present reviews about the conditions that the smart grid requires from communication point of view. Smart grid technologies and standards are investigated to come up with an outline of the smart grid model and the incorporation of various communication technologies in $[14,15]$. Some investigations stress on a particular standard or communication technology such as power line communication [16] and wireless communication $[17,18]$. In [19], the authors assess the network performance for a long-distance distribution line and propose communication layout for distribution level systems. Furthermore, the suitable communication technologies for transmission-level systems are discussed in [20].

Multiple-input multiple output (MIMO) techniques are being widely adopted in the current fourth generation $(4 \mathrm{G})$ telecommunication systems and they are expected to be a key technologies for the fifth-generation $(5 \mathrm{G})$ communications. MIMO systems take advantage of the multipath nature of the propagation channel. However, the antenna properties turn out to affect correlations among channel coefficients. MIMO, as a currently well established technology, offers considerable benefits, such as improving link quality and largely attainable data rates [21-28]. When antenna array elements are made closer one another, the effect of electromagnetic mutual coupling between them becomes a common phenomenon. The mutual coupling can dangerously deteriorate the performance of the array in the form of signal-to-interference-noise ratio (SINR) reduction and the signal processing algorithm non-convergence [29-30]. It precisely degrades some parameters such as the carrier frequency offset [31], channel [32], and angle of arrival estimations [33]. Also, the awful effect of mutual coupling on the active reflection coefficient of a MIMO antenna is another result that cannot be ignored [34]. Furthermore, the active voltage standing wave ratio (VSWR) may reach intolerable values. Despite the negative effects of mutual coupling the MIMO system performance deterioration, it can be exploited for array calibrations as in [35-36]. The mutual coupling modifies the antenna characteristics in an array, and hence affects the MIMO system performance (e.g., capacity, error rate, and spectral re-growth). The system performance can be partly improved by adjusting out the mutual coupling using digital techniques but without improving the SINR. Thus, it is very imperative to lessen the mutual coupling when it comes to MIMO antenna design. 
Optimization techniques have been widely employed in science and engineering to solve a variety of problems. This is due to the introduction of modern global non-classical techniques that are able to handle non-differentiable and non-linear complex functions along with the ever increasing processing power of computers. Genetic Algorithms (GA) [37-38], Differential Evolution (DE), and Particle Swarm Optimization (PSO) [39-40], are in the class of evolutionary techniques inspired from nature and constitute an illustrative example. Other recent techniques have also been deployed in antenna design including the Taguchi method [41-42] based on the concept of fractional factorial design and Orthogonal Arrays (OAs) that significantly reduce the number of iterations needed in the optimization process. The Galaxy-based Search Algorithm (GbSA) is another nature-inspired metaheuristic technique that imitates the behavior of spiral galaxies when searching its surroundings [28]. Metaheuristics are optimization algorithms having two fundamental aspects: exploitation and exploration. Exploration means a multitude of solutions is found within the search space to obtain the global optimum. In exploitation, local search exploits the information about the best solutions found so far. This blend along with selecting the best solutions will assure that the algorithm reaches optimality. Furthermore, the exploration avoids the local optima traps through randomization and increases the diversity of the solutions.

The literature includes many works regarding MIMO system antenna design optimization. Capacity formulas have been presented in [43] and [44] where mutual coupling and spatial correlation effects with a Rayleigh fading channel have been considered. The considered geometries in these studies comprise namely the uniformly excited linear arrays with spacing between the elements to be optimized. In [45] and [46], the right choice of the number of antennas at the asymmetric base station and a mobile unit has been addressed. In [47], MIMO system capacity optimization with different antenna installation costs at both MIMO ends has been considered. However, the cost function has been expressed using approximate formula for the ergodic capacity estimates. From geometry selection point of view, the Uniform Linear Array (ULA) is the most used geometry in the current wireless systems. The Uniform Circular array (UCA) is as another option as it exhibits some better properties. Recioui and Bentarzi [26-28] challenged capacity maximization via inter-element spacing determination in linear and 2D arrays considering the joint effects of mutual coupling and spatial correlation. A channel formula was proposed that englobes both mutual coupling and spatial correlation effects and has been compared with its separate counterparts. The results of this work show promising trends of system capacity enhancement compared to the studies in literature which can be beneficial in wireless communication system design.

In 2016, Mirjalili and Lewis [48] proposed the Whale Optimization Algorithm (WOA) to imitate the hunting activitiy of humpback whales. This activity is done by a combination of two main behaviors: randomly chasing the prey and the bubble-net hunting approach. Humpback whales possess an amazing hunting philosophy. This hunting behavior is known as the bubble-net feeding technique. Humpback whales are fond of hunting small fish flocks close to the ocean surface. So, they make in swimming around the target prey winding-shaped target pattern, producing distinguishing blebs along a circle or simply ' 9 ' shaped ways.

Several improvement initiatives of WOA have been done in the literature. These can be divided into two categories: improving the WOA's performance and Appling the WOA to solve some optimization problems. Some of WOA's improvement attempts are in [49-51]. Kaur and Arora [49] suggested chaotic WOA (CWOA) where the chaos of the ory are used for tuning the main parameters of WOA to enhance its rate of convergence. In [50], Lévy flight trajectory-based WOA (LWOA) is introduced. The LWOA employed Lévy flight trajectory to boost the population diversity. In [51], a modified WOA (MWOA) has been introduced to solve large scale global optimization problems. In MWOA the Lévy flight strategy was employed to improve WOA's exploration ability. To improve the exploitation ability of WOA, a quadratic interpolation method has been used in MWOA. In addition, MWOA utilized nonlinear control strategy to control the whole search process and balance the exploration and the exploitation ability of WOA. On the other hand, WOA has been applied to many real-world applications. In [52], the combination of 
WOA and pattern search (PS) algorithms [53] has been presented and applied to power system design. In [54], an approach of embedding simulated annealing (SA) into WOA to produce four feature selection methods has been proposed. SA was applied as a local search to improve the exploitation ability of WOA. Aljarah, Faris, and Mirjalili [55] applied WOA to Neural Networks training. In [56], WOA in conjunction with Moth-Flame Optimization (MFO) algorithms were applied to the multi-level thresholding image segmentation problem. In [57], WOA was utilized to tackle the optimal reactive power dispatch problem. Yu et al. [58] proposed levy flight WOA (LWOA) and used it to optimize the parameters of an active disturbance rejection control (ADRC) scheme for automatic carrier landing system (ACLS). In [59], Wu et al. improved WOA and utilized it for proposing a novel path planning framework for a solar-powered UAV (SUAV) in urban environment. WOA has been hybridized with DE which has good exploration ability and present an algorithm named Improved WOA (IWOA). The performance of IWOA has been improved by presenting an IWOA in which a new control parameter is introduced. In addition, re-initialization is integrated into IWOA to increase diversity of population.

In this paper, the WOA algorithm is employed to solve the problem of capacity maximization in MIMO systems cast as an optimization task. The various advantages of the whale optimization algorithm, such as the reduced number of parameters and lack of local optima entrapment constitute the motivation to this choice. Being dependent on the antenna array physical dimensions, the main goal is to find these physical dimensions at the transmitter/Receiver arrays that ensure the highest possible capacity. The MIMO systems considered in this work incorporate the conventionally used linear arrays along with the proposed 2D antenna arrays. The designed MIMO systems are tested on the wide area monitoring system employing phasor measurement units. The performance of the system in terms of data latency and completeness is compared with the currently used single input single output system.

\section{MIMO System Design Optimization}

\subsection{Problem formulation}

The literature defines the MIMO channel system capacity as: "the maximum data transfer rate (bits per second per hertz) for some acceptable level of received signal" and it is given in bps/Hz. Mathematically, the capacity of a MIMO channel having $\mathrm{Nt}$ transmitting antennas and $\mathrm{Nr}$ receiving antennas can be written as [13]:

$$
\operatorname{Cap}=\log _{2}\left[\operatorname{det}\left(I_{N_{r}}+\frac{S N R}{N_{t}} H H^{H}\right)\right]
$$

Where $\mathrm{I}_{\mathrm{Nr}}$ is an $\mathrm{N}_{\mathrm{r}}$-dimensional identity matrix, $\mathrm{SNR}$ is the mean received signal-to-noise ratio in $\mathrm{dB}$. $\mathrm{H}$ is the channel transfer matrix and the small $\mathrm{H}$ on the exponent denotes the complex conjugate transpose.

The channel matrix being random in nature, the capacity of the MIMO channel is then a random variable. Hence, the capacity measure of channels can be defined in various ways. Practically, two commonly used statistical measures: mean ergodic capacity and outage capacity are the most utilized. The mean capacity of a MIMO channel is defined as the ensemble average of the information rate over the all the realizations of the channel matrix [13]. The interpretation of the mean capacity is that in an ergodic channel, one can transmit the signal at the rate given by mean capacity without errors.

Mutual coupling effect is modelled within the channel transfer matrix written as [14-15]:

$$
H_{M C}=C_{R} G C_{T}
$$


Where $\mathrm{G}$ is an $N_{r}$-identity matrix whose entries are identically distributed (i.i.d) complex Gaussian zero-mean unit variance elements. $C_{R}$ and $C_{T}$ are the coupling matrices at the receiver and transmitter, respectively. These matrices can be computed for an array of half-wavelngth dipoles as:

$$
C=\left(Z_{A}+Z_{T}\right)\left(Z+Z_{T} I_{N}\right)^{-1}
$$

Where is the self impedance of the element in isolation $\left(Z_{A}=73+42.5 j \Omega\right.$ for a $\lambda / 2$ dipole; $\lambda$ is the wavelength). $Z_{T}$ is the impedance of the receiver at each antenna element taken to be the complex conjugate of $Z_{A}$ for impedance matching. $I_{N}$ is the identity matrix and $Z$ is an $N \times$ $N$ mutual impedance matrix that depends of the relative positions among the dipoles as shown in Fig.1.

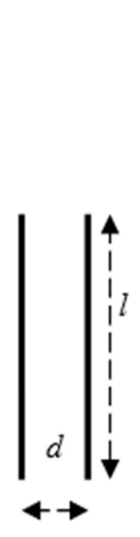

(a)

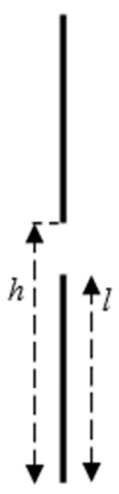

(b)

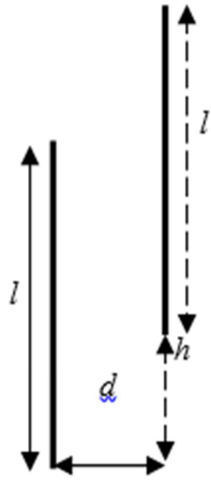

(c)

Fig 1. Relative positions of dipoles in an array (at Tx or Rx) for a linear array with their physical dimensions: (a) Side-by-side configuration; (b) Collinear configuration; (c) Parallel-in-echelon configuration.

Each of these arrangements has its closed form formulas that allow the calculation of the matrix $\mathrm{Z}$ and hence $\mathrm{C}_{\mathrm{T}}$ and $\mathrm{C}_{\mathrm{R}}$ of equations (2) and (3).

The channel matrix needs to be correctly normalized [15]. There exist two kinds of normalization: The first normalization is done on the each realization of the end-to-end channel gain as well as mutual coupling matrix $\mathrm{H}_{\mathrm{MC}}$, such that [15]

$$
\left\|H_{M C}\right\|_{F}^{2}=N_{r} \times N_{t}
$$

Where $\|.\|_{\mathrm{F}}^{2}$ is the Frobenius norm. The limitation of this normalization is that the disparities in the channel gain due to antennas are eliminated. However this type of normalization allows the investigation of the correlation between the channel matrix entries and gives good indication of the richness of the multipath environment [14-15].

The second normalization is summarized by the equation:

$$
\|G\|_{F}^{2}=N_{r} \times N_{t}
$$

This normalization is also performed on each realization of the channel matrix, but includes the propagation channel only. This normalization allows the investigation of the instantaneous effects of received power changes due to mutual coupling.

A 2D rectangular array may be regarded as a combination of individual linear arrays as shown in fig. 2. The mutual impedance matrices can be extended to the rectangular array. As shown in the example of fig. 2, the elements of the rectangular array exhibit the three configurations at the same time. As an illustration, element 1 (in bold) is in side-by-side configuration with the adjacent 
element(s), in collinear configuration with the element below and in parallel-in-echelon with the diagonal element.

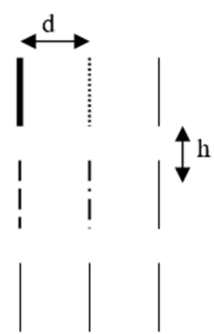

Fig 2. A $3 \times 3$ rectangular array with the physical dimensions.

Each element has mutual coupling with all the other elements in the rectangular array. Thus, they turn out to produce a $9 \times 9$ mutual impedance matrix in this example. Generally, an array with $N$ row elements and $M$ column elements results in a coupling matrix of size $M N \times M N$. The entries of the impedance matrix are computed based on the equations in $[28,63]$ with respect to the relative arrangement of the elements. The impedance matrix in this example can be represented as:

$$
Z=\left[\begin{array}{lll}
Z_{11} & Z_{12} & Z_{13} \\
Z_{21} & Z_{22} & Z_{23} \\
Z_{31} & Z_{32} & Z_{33}
\end{array}\right]
$$

Where the entries given above should be viewed as $3 \times 3$ block matrices. The matrix is symmetrical about its diagonal block elements. The diagonal block elements represent the mutual impedance matrices within the horizontal arrays. These are computed based on the side by side configurations. The off-diagonal block matrices are computed depending on the relative positions of the elements. As an example, $Z_{12}$ is the $3 \times 3$ impedance matrix due to the array 1 and array 2 . The diagonal elements of this matrix represent the mutual impedance due to the elements in array 2 with those just above them. These are in collinear configuration. The other entries are computed using the parallel-in-echelon configuration. Finally, the other blocks are just constructed by symmetry. With the impedance matrix computed, the coupling matrix is determined using (3) and hence the system capacity is obtained using (1) and (2).

\subsection{The whale optimization algorithm}

\subsubsection{Underlying background}

Whale Optimization Algorithm (WOA) is a meta-heuristic optimization algorithm that considers the hunting behavior of humpback whalesas a model. This algorithm situates itself as a different technique compared to Grey Wolf Optimization [60]. It Imitates the hunting strategy to catch the prey and the use of a spiral to simulate the bubble-net attacking mechanism of humpback whales.

Whales possess cells in certain areas of their brains similar to those of human named spindle cells. These cells are responsible of judgment, emotions, and social behaviors. The number of these cells is twice compared an adult human which justifies their cleverness. The inter-relationship of whales is another amazing property. They are found to be able to stay lonely or may live in groups. However, in most of the time, they are found in groups.

Of the largest whales, one would list the humpback whales. The most attractive feature of the humpback whales is their exceptional hunting technique. Their chasing mechanism is named bubble-net feeding technique [61]. Humpback whales are keen to hunt group of krill or small fishes close to the ocean surface. This foraging strategy is performed by generating specific bubbles along

Youcef Grainat et al (Application and Optimization of MIMO Communication in Wide Area Monitoring Systems) 
a circle or ' 9 '-shaped pattern as Fig. 3 illustrates. In this patten, humpback whales dive down for about $12 \mathrm{~m}$ and then start to produce bubbles in a spiral manner around the prey and swim up towards the surface of the ocean. The latter mechanism includes three distinct stages: coral loop, lobtail, and capture loop. Bubble-net feeding is specific to humpback whales. This spiral bubble-net feeding strategy has been mathematically represented in order to be useful as an optimization procedure [48].

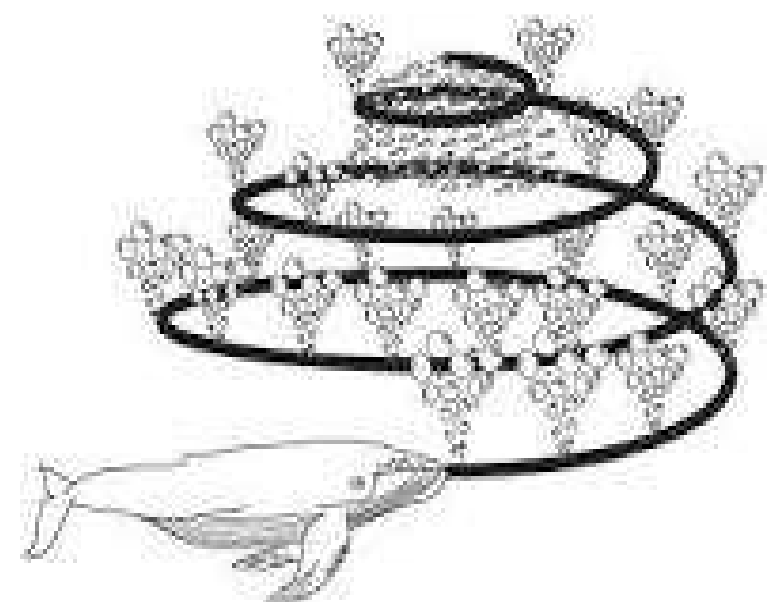

Fig 3. Whale Bubble-Net feeding strategy

\subsubsection{Presentation of the WOA:}

As it is the case in all other techniques, the WOA algorithm initiates with random solutions. At each iteration, these solutions evolve byupdating their positions. The customized evolution of the solution set makes the WOA swap easily between exploration and exploitation. In addition, WOA has mainly two major internal parameters to be set. WOA possess a high exploration ability owing to the Whales position evolution process. In WOA, high exploitation and convergence are overstressed. The mathematical procedure reveals that the WOA algorithm cannot only avoid getting trapped at local optima avoidance but ensures high convergence speed during the optimization process.

The algorithm involves two major parts: the first consists in prey encirclment and the spiral position update (exploitation phase). In the second, a random Search for the prey is done (exploration phase) [48]. The course of these phases is described mathematically in the following subsections.

\subsubsection{Prey Encirclment}

In this step, humpback whale determines the prey position and starts the encirclment. The initialization of search variables is strictly random over the search-space. Hence, WOA considers the best found position as target prey location. The remaining candidate solutions must update their positions in accordance to this target prey location. This step is reptesented mathematically as [48]:

$$
\begin{aligned}
& \vec{D}=\left|\vec{C} \cdot \overrightarrow{X_{\text {best }}(t)}-\overrightarrow{X(t)}\right| \\
& \overrightarrow{X(t+1)}=\overrightarrow{X_{\text {best }}(t)}-\vec{A} \cdot \vec{D}
\end{aligned}
$$

Where $\vec{X}$ is the current solution set, $\overrightarrow{X_{\text {best }}}$ is the best solution found so far, $t$ means the current iteration. The values of $\vec{A}$ and $\vec{C}$ are mathematically found as: 


$$
\left\{\begin{array}{c}
\vec{A}=2 \vec{a} \cdot \overrightarrow{r_{1}}-\vec{a} \\
\vec{C}=2 \vec{a} \cdot \overrightarrow{r_{2}}
\end{array}\right.
$$

Where $\overrightarrow{r_{1}}$ and $\overrightarrow{r_{2}}$ are two random vectors taken in $(0,1) ; \vec{a}$ is the search direction matrix chosen to be linearly decreasing from 2 to 0 throughout the course of optimization. In (10), $\vec{D}_{i}^{\text {new }}=$ $\left|\overrightarrow{X_{\text {best }}(t)}-\overrightarrow{X(t)}\right|$ is the distance between $i^{t h}$ whale to prey; $\mathrm{b}$ is a constant; $\mathrm{r}_{3}$ is a random number in the range $(-1,1)$.

$$
\overrightarrow{X(t+1)}=\vec{D}_{i}^{\text {new }} e^{b r_{3}} \cos \left(2 \pi r_{3}\right)+\overrightarrow{X_{\text {best }}(t)}
$$

It is remarked that humpback whales swim around the prey around a decreasing-radius circle and following a spiral trajectory concurrently. The mathematical model of this stage is represented as:

$$
\overrightarrow{X(t+1)}= \begin{cases}\overrightarrow{X_{\text {best }}(t)}-\vec{A} \cdot \vec{D} & \text {;if } p<0.5 \\ \vec{D}_{i}^{\text {new }} e^{b r_{3}} \cos \left(2 \pi r_{3}\right)+\overrightarrow{X_{\text {best }}(t)} ; \text { if } p \geq 0.5\end{cases}
$$

where $\mathrm{p}$ is a random number in $(0,1)$.

\subsubsection{Bubble-Net Feed}

The bubble-net feeding strategy was modeled based on two mechanisms as in [48]:

- Decreasing-radius encirclement process

This is done by decreasing $\vec{a}$ from 2 to 0 during the progress of the optimization task. This step allows also the reduction in the oscillations of $\vec{A}$.

\section{- Spiral update strategy}

To be able to establish a spiral trajectory for Search for prey (exploration), $\vec{A}$ is varied in the interval $(-1,1)$ to search for prey. The Humpback whales chase the prey randomly with respect to the position of each other. The positions of whales are updated with respect to a randomly chosen whale rather than that of the best whale found so far. Mathematically, this exploration is represented as:

$$
\left\{\begin{array}{l}
\vec{D}=\left|\vec{C} \cdot \overrightarrow{X_{p}(t)}-\overrightarrow{X(t)}\right| \\
\overrightarrow{X(t+1)}=\overrightarrow{X_{p}(t)}-\vec{A} \cdot \vec{D}
\end{array}\right.
$$

Where $\overrightarrow{X_{p}(t)}$ is the random location of whales. The general flowchart of WOA is shown in Fig. 4. 


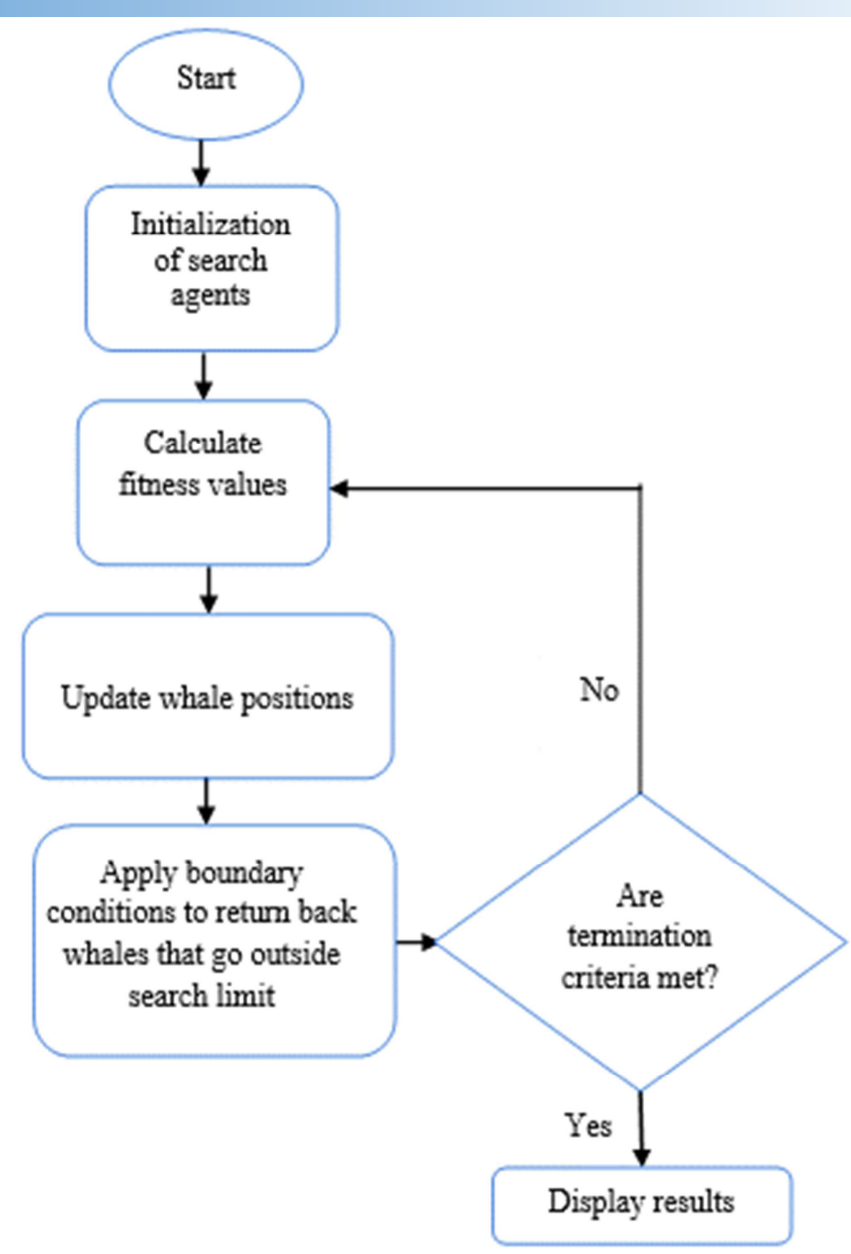

Fig 4. Flowchart of WOA

\subsection{Optimization Results and discussions}

In this optimization task, various MIMO systems employing linear and rectangular antenna arrays at Tx and Rx are considered. For all the systems, the average SNR is fixed to $20 \mathrm{~dB}$. Concerning the array inter-element separations, both horizontal spacing (d) and vertical spacing (h) are confined in the interval $0.25 \kappa$ to $2 \lambda$; where is the operating wavelength. The optimization task for linear arrays is performed following a similar approach to [28]. For rectangular arrays and to reduce time and space complexities, the array size on either dimension does not exceed 3 . Table 1 summarizes the optimized capacity values for the $2 \mathrm{D}$ rectangular arrays along with the linear arrays. One would clearly see from Table 1 that the capacity values produced by the WOA outperform the ones of uniform linear and 2D arrays.

Various examples are dealt with to illustrate the need for optimization and to justify the deployment of 2D arrays at Tx and Rx. For the linear array case, it can be easily seen that the nonuniform arrays promise better capacity values than those when uniform arrays are deployed.

As far as the 2D arrays are concerned, the first illustration considers $2 \times 2$ rectangular arrays used at both ends taking into account uniform and non-uniformly spaced cases. The results show that a capacity improvement compared to the uniform linear and 2D cases. As a matter of fact, an improvement of $7.2 \%$ in capacity value is noticed with respect to the $3 \times 3$ uniform linear array case as it is the largest linear array size. 
In the next example, a $3 \times 2$ rectangular array is employed at the transmitter with a $2 \times 2$ array at the receiver. The results show a further capacity enhancement than a 3 -element linear array deployed at both ends. The capacity increase is found to be $8.7 \%$ relative to the $2 \mathrm{D}$ uniform array.

As a third illustration, a $3 \times 2$ rectangular array has been deployed at the Rx and a $2 \times 2$ one at the Tx. This case resulted in a capacity improvement of $10 \%$ compared to the employment of 3dimensional uniform linear arrays and $2.65 \%$ with respect to the uniform $2 \mathrm{D}$ array. As a last example, both ends are equipped with $3 \times 3$ rectangular arrays. The non-uniform rectangular array produces a capacity value that is $7 \%$ more than when using even a 4-dimensional uniform linear array and $45.85 \%$ relative to using 3 -element uniform linear arrays. It is worth to mention that in the linear array case; the only the dimension considered here is the horizontal because of the use of the side-by-side arrangement.

On the other hand, one very important advantage of favoring the employment of non-uniform arrays is that it is possible to obtain better performance (in terms of capacity) with a reduced array size. This is beneficial in the sense that the space occupied by this reduced size array is less than its uniform counterpart which makes it of practical use. Another remark is that the use of the two dimensional arrays has led to a further enhancement in capacity witnessed by the values which overtake the ones obtained when optimization one dimensional arrays. It can be remarked that rectangular arrays promise a larger capacity value than linear arrays with even larger dimension. $3 \times 3$ rectangular arrays at both ends would perform better than 4-element linear arrays. Though the space occupied would be larger, the gain in capacity makes it worth it to employ rectangular arrays.

In fact, non-uniform linear arrays promise an increase in capacity that can go up to $20 \%$ over uniform ones and the employment of rectangular arrays produce up to $35 \%$ over the currently employed uniform linear arrays. One explanation of the results comes directly from equation (1) where the channel matrix $H$ becomes highly scattered as rectangular arrays are employed. This leads directly to an increase in the system capacity as more routes are available for the data to be transmitted reliably over the MIMO channel.

Table 1. Results of capacity optimization for different MIMO Systems

\begin{tabular}{|c|c|c|c|c|}
\hline Array Type & System $\mathbf{N}^{\circ}$ & Dimensions & $\begin{array}{c}\text { Uniform Capacity } \\
\text { (reported in [32] for linear } \\
\text { and in }[28,62] \text { for } 2 \mathrm{D}) \\
(\mathrm{bps} / \mathrm{Hz})\end{array}$ & $\begin{array}{l}\text { WOA Optimized } \\
\text { capacity (bps/Hz) }\end{array}$ \\
\hline \multirow{4}{*}{ Linear } & 1 & Tx: 2 |Rx: 2 & 12.625 & 13.23 \\
\hline & 2 & Tx: $2 \mid R x: 3$ & 13.54 & 14.19 \\
\hline & 3 & Tx: 3 |Rx: 2 & 13.79 & 15.06 \\
\hline & 4 & Tx: 3 |Rx: 3 & 17.23 & 17.65 \\
\hline \multirow{4}{*}{ 2D } & 5 & Tx:2×2|Rx: $2 \times 2$ & 17.91 & 18.47 \\
\hline & 6 & Tx: $3 \times 2 \mid R x: 2 \times 2$ & 18.34 & 18.73 \\
\hline & 7 & Tx: $2 \times 2 \mid R x: 3 \times 2$ & 18.47 & 18.95 \\
\hline & 8 & Tx: $3 \times 3 \mid \operatorname{Rx}: 3 \times 3$ & 24.65 & 25.13 \\
\hline
\end{tabular}




\section{MIMO Systems in Wide Area Monitoring}

\subsection{System description}

The work emulates a small scale wide area monitoring system. The power system considered in this work is the IEEE 14-bus standard system. Previous works have shown that with one zero injection at bus 7, this system can be made completely observable using only 3 PMUs placed at buses 2,6 and 9. Fig. 5 represents the MATLAB SIMULINK model of the simulated system. The raw data collected from the three PMUs is sent through both the SISO channel and the MIMO channel. To account for the delays, the three PMUs are assumed to be located at different distances from the PDC. This is translated into different delays introduced into the set data. The three PMUs are synchronized using the GPS clock. In the SISO channel, the data is sent as a stream while in the MIMO channel; data is splitted into two streams (sent at two antennas) and is combined back at the receiver. Due to the fact that data arrives at the receiver from different directions, maximum combining is used to ensure that the received data undergoes the minimum possible error. The MIMO channel is assumed to exhibit a Rayleigh fading which is close to reality. To assess the performance of both systems, two performance indicators are considered: the time of data flow through the channel to account for the data latency and the bit error rate to account for data completeness. It should be noted that the state of the power system state is estimated using the received data from the three PMUs.

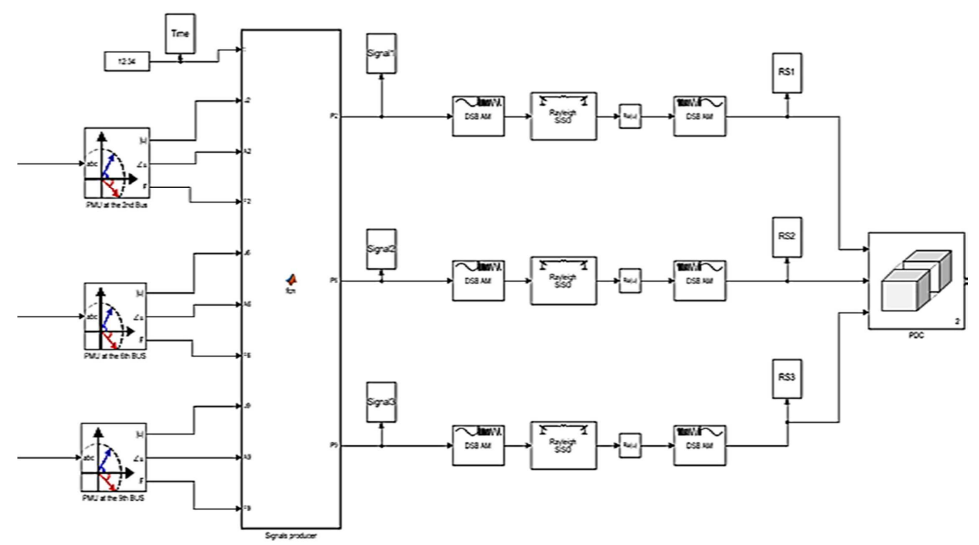

Fig 5. Simulink model of SISO system

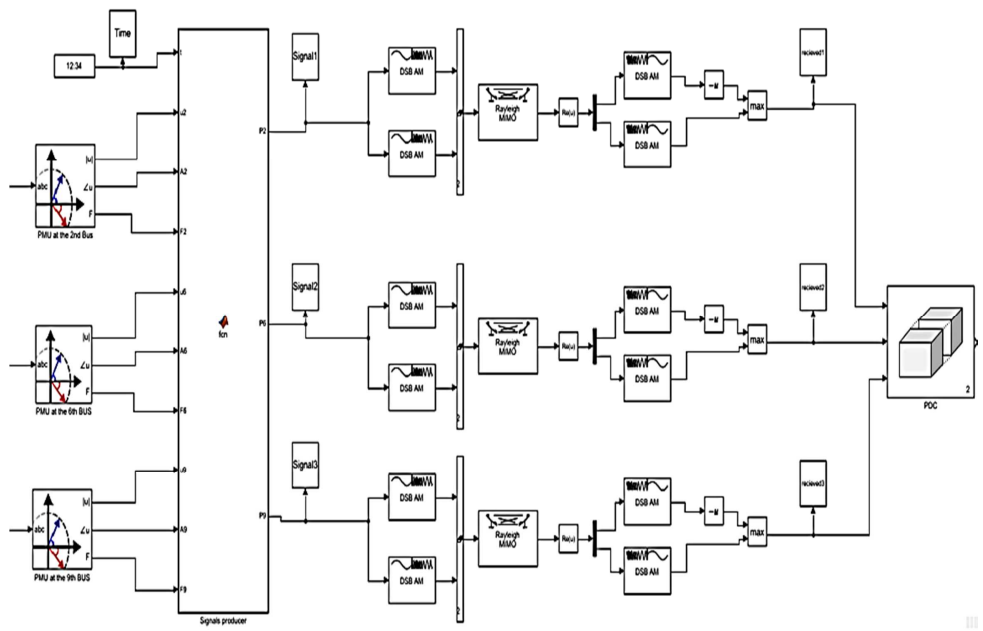

Fig 6. Simulink model of MIMO system 


\subsection{Results and discussions}

Figure 5 and Figure 6 show the SISO and MIMO implementations of the previously defined systems, respectively. The same amount of data is sent over the SISO and MIMO systems. At the receiver level, the data is assessed for completeness with reference to the sent data and latency as measured by the SIMULINK counter for both systems. The Total Vector Error (TVE) is taken as measure of date completeness.

Table 2. Performance measures of both SISO and MIMO systems

\begin{tabular}{|c|c|c|c|c|}
\hline Array Type & Dimensions & Bus Number & Delay (latency-ms) & $\begin{array}{c}\text { Completeness (TVE- } \\
\%)\end{array}$ \\
\hline \multirow{3}{*}{ SISO } & \multirow{3}{*}{$\begin{array}{l}\text { Tx: } 1 \\
\text { Rx: } 1\end{array}$} & 2 & 1.93 & 1.3 \\
\hline & & 6 & 1.53 & 1.535 \\
\hline & & 9 & 1.03 & 1.515 \\
\hline \multirow{12}{*}{$\begin{array}{l}\text { MIMO } \\
\text { (Linear } \\
\text { arrays) }\end{array}$} & \multirow{3}{*}{$\begin{array}{l}\text { Tx: } 2 \\
\text { Rx: } 2\end{array}$} & 2 & 4.28 & 0.850 \\
\hline & & 6 & 3.15 & 1.150 \\
\hline & & 9 & 2.19 & 1.050 \\
\hline & \multirow{3}{*}{$\begin{array}{l}\text { Tx: } 2 \\
\text { Rx: } 3\end{array}$} & 2 & 4.28 & 0.850 \\
\hline & & 6 & 3.15 & 1.150 \\
\hline & & 9 & 2.19 & 1.050 \\
\hline & \multirow{3}{*}{$\begin{array}{l}\text { Tx: } 3 \\
\text { Rx: } 2\end{array}$} & 2 & 4.22 & 0.840 \\
\hline & & 6 & 3.21 & 1.100 \\
\hline & & 9 & 2.31 & 1.020 \\
\hline & \multirow{3}{*}{$\begin{array}{l}\text { Tx: } 3 \\
\text { Rx: } 3\end{array}$} & 2 & 4.75 & 0.775 \\
\hline & & 6 & 3.36 & 1.040 \\
\hline & & 9 & 2.34 & 0.975 \\
\hline \multirow{12}{*}{$\begin{array}{c}\text { MIMO } \\
\text { (2D arrays) }\end{array}$} & \multirow{3}{*}{$\begin{array}{l}\text { Tx: } 2 \times 2 \\
\text { Rx: } 2 \times 2\end{array}$} & 2 & 4.86 & 0.750 \\
\hline & & 6 & 3.76 & 1.050 \\
\hline & & 9 & 2.71 & 0.950 \\
\hline & \multirow{3}{*}{$\begin{array}{l}\mathrm{Tx}: 3 \times 2 \\
\mathrm{Rx}: 2 \times 2\end{array}$} & 2 & 4.41 & 0.710 \\
\hline & & 6 & 3.26 & 0.970 \\
\hline & & 9 & 2.36 & 0.905 \\
\hline & \multirow{3}{*}{$\begin{array}{l}\mathrm{Tx}: 2 \times 2 \\
\mathrm{Rx}: 3 \times 2\end{array}$} & 2 & 4.25 & 0.700 \\
\hline & & 6 & 3.29 & 0.955 \\
\hline & & 9 & 2.41 & 0.925 \\
\hline & \multirow{3}{*}{$\begin{array}{l}\text { Tx: } 3 \times 3 \\
R x: 3 \times 3\end{array}$} & 2 & 4.64 & 0.625 \\
\hline & & 6 & 3.47 & 0.835 \\
\hline & & 9 & 2.42 & 0.820 \\
\hline
\end{tabular}


Table 2 summarizes the performance indicators for both systems for comparison. It is clearly seen that the MIMO system performs better than the SISO systems in terms of data completeness. This is due to the fact that MIMO systems are mainly introduced to cure the impairments of the channel. Furthermore, the signal reception at the receiver is done as so to minimize the Total Vector Error (TVE). However, the SISO system is better in terms of latency as the data is a single stream and it is traveling a single channel towards the receiver while in the MIMO system, data is splitted and is scattered in the channel and combined back at the receiver. Hence, the designer has to compromise between the two performance measures when is comes to practical system design.

The IEEE C37.118.1a Standard provides the bounds of the PMU reporting latency. Also, this standard [63] specifies a much stricter Synchrophasor method and provides an error model for vectors. The Total Vector Error (TVE) needs to be maintained below 1\%. The results show that the MIMO system well respects the requirements of the standard in terms of data completeness. For the latency, it is found that it depends on the chosen reporting rate. The Standard provides two different limits for the maximum reporting latency of the two performance classes, depending on the reporting rate (RR) [64]. The reporting rate that can take values of 10, 25 and 50 frames/s for a system rated frequency of $50 \mathrm{~Hz}$ (lower reporting rates and, in particular, a reporting rate of 1 frame/s are possible within the PMU settings). The P class, specific for protection applications, has the limit (in seconds) equal to $\frac{2}{R R}$. The $\mathrm{M}$ class, specific for measurement applications, has the limits relaxed to $\frac{7}{R R}$, thus allowing the algorithms to work with a higher number ofcycles in order to obtain a more accurate measurement result.

It should be noted that MIMO systems are introduced mainly to cope with the limited power and bandwidth. So, a MIMO system promises more data to be put onto the communication system and hence the system would use more data and incorporate many applications simultaneously. In a wide area system, data can be used for monitoring, control and protection using the same data package. Furthermore, the study made here aims at highlighting the promising applicability of the MIMO communication systems into mart grid applications. A complete design of the MIMO system itself must be done to improve further the whole system. This includes the transmitter/Receiver architectures, the channel models and the modulation/coding schemes. Hence, the Smart grid application and the MIMO communication system constitute a complex system that needs to be optimized for performance at all levels.

\section{Conclusion}

The communication infrastructure is considered to have amajor role in the success of smart gridimplementation campaigns. The purpose of this work was to illustrate the potential benefits that acommunication system designer within the framework of smartgrid would gain when opting for a MIMOcommunication link. It has been shown that for the sameapplication, a MIMO system outperforms its SISOcounterpart in terms of data completeness which is a majorissue as all decisions and control actions resultsfrom correct data reception. Also, It has been that the MIMOsystem is relatively sluggish compared to theSISO system. This issue can be addressed by consideringmore details of the MIMO system design. On theother hand, the MIMO system promises higher data capacity tobe injected into the system which makes itbeneficial as many smart grid applications can be consideredsimultaneously. It should be noted that the smartgrid system using MIMO communication is very complex andopens the window to an optimization task thathas to be done on both the application itself and the MIMO communication system. 


\section{Nomenclature}

$\begin{array}{ll}\text { Cap } & \text { Channel capacitybps/Hz } \\ \mathrm{SNR} & \text { Signal to Noise Ratiodimensionless } \\ \mathrm{N} & \text { Nbr of elements in the transmitter } \\ \mathrm{N}_{\mathrm{r}} & \text { Nbr of elements in the reciever } \\ \mathrm{Z} & \text { The impedence Ohm } \\ \text { TVE } & \text { Total Vector Error \% }\end{array}$

Greek letters

$\lambda$

The wavelength $\mathrm{m}$

Subscripts

bps $/ \mathrm{Hz} \quad$ Bit per second per Hertz.

$\mathrm{ms}$

Milliseconds.

m Meter

Nbr Number

\section{Acknowledgment}

This work has been conducted under the national research project $\mathbf{N}^{\circ}: \mathbf{A 0 1 L 0 7 U N 3 5 0 1 2 0 1 8 0 0 0 2}$ Supported by "la Direction Générale de la Recherche Scientifiqueet du Développement Technologique (DGRSDT)" inAlgeria. The authors would like also to thank Prof. Hamid BENTARZI; the head of the signals and systems laboratory forhis support towards the achievement of this work.

\section{References}

[1] Kuzlu, M.; Pipattanasomporn, M.;Saifur, R. Communicationnetwork requirements for major smart grid applications in HAN, NAN and WAN. Computer Networks 2014, 67, 74-88.

[2] Miao, L.; Wei, G.; Fang, X.; Risheng, J. The strategy of the voltage control in smart grid based on modern control method and FPGA. Proceedings of 34th Chinese Control Conference (CCC), 2015, 8964-8968.

[3] Gellings, C. W. The concept of demand-side management for electric utilities. Proceedings of IEEE, 1985, 73(10), 1468-1470.

[4] Tsai, C.W.; Pelov, A.; Chiang, M.C.; Yang, C.-S.; Hong, T.P. Computational awareness for smart grid: a review. Int. J. Mach. Learn. Cybern. 2014, 5(1),151-163.

[5] Farhangi, H. The path of the smart grid. IEEE Power Energy Mag. 2010,8(1),18-28.

[6] Park, N.; Kim, M. Implementation of load management application system using smart grid privacy policy in energy management service environment. Clust. Comput. 2014,17(3), 653-664.

[7] Recioui, A.; Bentarzi, H.; Tsebia, M. PMU Deployment in Power System Oscillation Monitoring. In Sustainable aviation 2014, 312: 322.

[8] Momoh, J. Smart Grid:Fundamentals of Design and Analysis. 2012.

[9] C37.118-2005, IEEE Standard for Synchrophasors for Power Systems, 2006.

[10] Dolezilek, D.; Fischer, N.; Schloss, R. Improvements in Synchronous Wide-Area Data Acquisition Design and Deployment for TelecontrolandTeleprotection,

2012. <https://www.selinc.com/WorkArea/ DownloadAsset.aspx?id=99365>.

[11] Yan, Y.; Qian, Y.; Sharif H.; Tipper, D. A Survey on Smart Grid Communication Infrastructures: Motivations, Requirements and Challenges. IEEE Communications Surveys \& Tutorials 2013,15(1), 5 - 20. DOI: 10.1109/SURV.2012.021312.00034.

[12] Wang, W.; Xu, Y.; Khanna, M. A survey on the communication architectures in smart grid. Comput. Netw 2011, 55, 3604-3629.

[13] Khan, R.H.; Khan, J.Y. A comprehensive review of the application characteristics and traffic requirements of a smart grid communications network. Comput. Netw 2013, 57, 825-845. 
[14] Gungor, V.C.; Sahin, D.; Kocak, T.; Ergut, S.; Buccella, C.; Cecati, C.; Hancke, G.P. Smart grid technologies: communication technologies and standards. IEEE Trans. Indu. Inform. 2011, 7 (4), 529-539.

[15] Fan, Z.; Kulkarni, P.; Gormus, S.; Efthymiou, C.; Kalogridis, G.; Sooriyabandara, M.; Zhu, Z.; Lambotharan, S.; Chin, W. Smart grid communications: overview of research challenges, solutions, and standardization activities. IEEE Commun. Surv. Tutorials 2012, 99, 1-8.

[16] S. Galli, A. Scaglione, Z. Wang, Power line communications and the smart grid, in: IEEE International Conference Smart Grid Communications (SmartGridComm), 2010, pp. 303-308.

[17] Pipattanasomporn, M.; Kuzlu, M.; Rahman, S. Demand response implementation in a home area network: a conceptual hardware architecture, In IEEE Innovative Smart Grid Technologies (ISGT) Conference, 2012, $1-8$.

[18] Wietfeld, C.; Georg, H.; Groening, S.; Lewandowski, C.; Mueller, C.; Schmutzler, J. Wireless M2M communication networks for smart grid applications. In Sustainable Wireless Technologies (European Wireless), 2011, 1-7.

[19] Aravinthan, V.; Karimi, B.; Namboodiri, V.; Jewell, W. Wireless communication for smart grid applications at distribution level -feasibility and requirements. In IEEE Power and Energy Society General Meeting $2011,1-8$

[20] Dong, Y.; Kezunovic, M. Communication infrastructure for emerging transmission-level smart grid applications. In IEEE Power and Energy Society General Meeting 2011, 1-7.

[21] Jianfeng, L.; Defu, J.; Xiaofei, Z. DOA Estimation Based on Combined Unitary ESPRIT for Coprime MIMO Radar. IEEE Communications Letters 2017,21(1), 96-99.

[22] Said, M.M.; Yahia, M.M.A. On cross correlation in antenna arrays with applications to spatial diversity and MIMO systems. IEEE Transactions on Antennas and Propagation 2015, 63(4), 1798 - 1810.

[23] Sebastien, C.; Said, M.M.; Yahia, M.M.A. A generalized methodology for obtaining antenna array surface current distributions with optimum cross-correlation performance for MIMO and spatial diversity applications. IEEE Antennas and Wireless Propagation Letters 2015,14, 1451 - 1454.

[24] Wojciech, J. K. Space diversity parameters of MIMO systems small antenna array for mobile terminal. In Proc. European Conference on Antennas and Propagation, Davos, Switzerland 2016, 1-4.

[25] Foschini, G. J.; Gans, M. J. On Limits of Wireless Communications in a Fading Environment When Using Multiple Antennas. Wireless Personal Communications 1998, 6(3), 311-335.

[26] Recioui, A.; Bentarzi, H. Genetic Algorithm based MIMO capacity enhancement in spatially correlated channels including Mutual Coupling. Wireless Personal communications 2012, 63(3), 689-701.

[27] Recioui, A.; Bentarzi, H. Capacity Optimization of MIMO Wireless Communication Systems Using a Hybrid Genetic-Taguchi Algorithm. Wireless Personal Communications 2013, 71(2), 1003-1019.

[28] Recioui, A.; Bentarzi, H. Application of a Galaxy-Based Search Algorithm to MIMO System Capacity Optimization. Arabian Journal for Science and Engineering 2016, 41(9), 3407-3414.

[29] Yuan, Q.; Chen, Q.; Sawaya, K. Performance of adaptive array antenna with arbitrary geometry in the presence of mutual coupling. IEEE Trans. Antennas Propag. 2006, 54(7), 1991-1996.

[30] Wang, B; Chang, Y.; Sun, Y. Performance of the large-scale adaptive array antennas in the presence of mutual coupling. IEEE Trans. Antennas Propag. 2016, 64(6), 2236-2245.

[31] Wu, Y.; Bergmans, J. W. M.; Attallah, S. Effects of antenna correlation and mutual coupling on the carrier frequency offset estimation in MIMO systems. In Int. Conf. Wireless Commun. Netw. Mobile Computing (WiCOM), Chengdu, China, 23-25 Sept. 2010.

[32] Lu, S. ; Hui, H. T. ;Bialkowski, M. E. et al. The effect of antenna mutual coupling on channel estimation of MIMO-OFDM systems. IEEE Antennas Propag. Society. In Int. Symp., Honolulu, HI, Jun. 2007, 1-4.

[33] Lui, H. S.; Hui, H. T. Mutual coupling compensation for direction-of-arrival estimations using the receivingmutual impedance method. Int. J. Antennas Propag. 2010, 1-7.

[34] Pozar, D. M. A relation between the active input impedance and the active element pattern of a phased array. IEEE Trans. Antennas Propag. 2003, 51(9), 2486-2489. 
[35] Aumann, H. M.;Fenn, A. J.;Willwerth, F. G. Phased array antenna calibration and pattern prediction using mutual coupling measurements. IEEE Trans. Antennas Propag. 1989, 37(7), 844-850.

[36] Wei, H.; Wang, D.; Zhu, H. et al. Mutual coupling calibration for multiuser massive MIMO systems. IEEE Trans.WirelessCommun. 2016,15(1), 606-619.

[37] Recioui, A.; Azrar, A. Use of Genetic Algorithms in Linear and Planar Antenna. Microwave And Optical Technology Letters 2007, 49(7).

[38] Recioui, A.; Azrar, A.; Bentarzi, H., Dehmas, M.; Challal, M. Synthesis of Linear Arrays with SidelobeLevel Reduction Constraint using Genetic Algorithms. International journal of microwave and optical technology 2008, 3(5).

[39] Khodier, M. M.; Christodoulou, C. G. Linear array geometry synthesis with minimum side lobe level and null control using particle swarm optimization. IEEE Trans. on Antennas Propagat. 2005, 53(8), 2674-2679.

[40] Recioui, A. Sidelobe Level Reduction in Linear Array Pattern Synthesis Using Particle Swarm Optimization. J. of Optimization Theory and Applications 2012, 153(2), 497-512. DOI 10.1007/s10957-011-9953-9.

[41] Dib, N.; Goudos, S.; Muhsen, H. Application of taguchi's optimization method and selfadaptive differential evolution to the synthesis of linear antenna arrays. PIER 2010, 102, 159-180.

[42] Recioui, A. Optimization of Antenna Arrays Using Different Strategies Based on Taguchi Method. Arabian Journal for Science and Engineering 2014, 39(2), 935-944.

[43] Durrani, S.; Bialkowski, M. E. Effect of mutual coupling on the interference rejection capabilities of linear and circular arrays in CDMA systems. IEEE Trans. Antennas Propagat. 2004,52(4),1130- 1134.

[44] Piazza, D.; Kirsch, N. J.; Forenza, A.; Heath, R. W.; Dandekar, K. R. Design and evaluation of a reconfigurable antenna array for MIMO systems. IEEE trans. Anten. Propag. 2008, 56(3).

[45] Lozano, A.; Tulino, A. M. Capacity of multipletransmit multiple receive antenna architectures. IEEE Trans. Inf. Theory 2002,48(12), 3117-3127.

[46] Oyman, O.; Nabar, R. U.; Bolcskei, H.; Paulraj, A. J. Tight lower bounds on the ergodic capacity of Rayleigh fading MIMO channels. In Proc. GLOBECOM, Taipei, Taiwan, R.O.C., Nov. 2002, 1172-1176.

[47] Du, J.; Li, Y. Optimization of antenna configuration for MIMO systems. IEEE transactions on Communications 2005,53(9), 1451-1454.

[48] Mirjalili, S.; Lewis, A. The whale optimization algorithm. Advances in Engineering Software 2016, 95, 51-67.

[49] Kaur, G.; Arora, S. Chaotic whale optimization algorithm. Journal of Computational Design and Engineering 2017.

[50] Ling, Y.; Zhou, Y.; Luo, Q. Lévy flight trajectory-based whale optimization algorithm for global optimization. IEEE Access 2017, 5, 6168-6186.

[51] Sun, Y.; Wang, X.; Chen, Y.; Liu, Z. A modified whale optimization algorithm for large-scale global optimization problems. Expert Systems with Applications 2018, 114, 563-577.

[52] Bentouati, B. ; Chaib, L. ;Chettih, S. A hybrid whale algorithm and pattern search technique foroptimalpower flow problem. In 2016 8th International Conference on Modelling, Identification and Control (ICMIC), Algiers, 1048-1053.

[53] Findler, N. S. V.; Lo, C.; Lo, R. Pattern search for optimization. Mathematics and Computers in Simulation 1987, 29(1), 41-50.

[54] Mafarja, M. M.;Mirjalili, S. Hybrid Whale Optimization Algorithm with simulated annealing for feature selection. Neurocomputing 2017, 1-11.

[55] Aljarah, I.; Faris, H.; Mirjalili, S. Optimizing connection weights in neural networks using the whale optimization algorithm. Soft Computing 2018, 22(1).

[56] Aziz, M. A. E.; Ewees, A. A. ; Ella, A. Whale Optimization Algorithm and Moth-Flame Optimization for multilevel thresholding image segmentation. Expert Systems with Applications 2017, $83,242-256$.

[57] Ben oualidMedani, K.; Sayah, S.;Bekrar, A. Whale optimization algorithm based optimal reactive power dispatch: A case study of the Algerian power system. Electric Power Systems Research 2018, 163, 696-705. 
[58] Yu, Y. ; Wang, H. ; Li, N. ; Su, Z. ; Wu, J. Automatic carrier landing system based on active disturbance rejection control with a novel parameters optimizer. Aerospace Science and Technology 2017, 69, 149-160.

[59] Wu, J.; Wang, H.; Li, N.; Yao, P.; Huang, Y.; Yang, H. Path planning for solar-powered UAV in urban environment. Neurocomputing 2018, 275, 2055-2065.

[60] Mirjalili S.; Mirjalili, S.M.; Lewis, A. Grey wolf optimizer. AdvEngSoftw. 2014, 69, 46-61.

[61] Watkins, W.A.; Schevill, W.E. Aerial observation of feeding behavior in four baleen whales: Eubalaenaglacialis, Balaenoptera borealis, Megapteranovaean-gliae, and Balaenopteraphysalus. J Mammal 1979,155-63.

[62] Recioui, A. Use of Spiral Optimization Technique to Enhance the Capacity of MIMO Communication Systems Employing One and Two-Dimensional Array Antennas. In International conference on applied analysis and mathematical modelling, Yildiz Technical university, Istanbul, Turkey, June 8-12, 2015.

[63] A. Phadke and R. de Moraes. The Wide World of Wide-area Measurement. Power and Energy Magazine, IEEE,6(5):52-65, Sep-Oct 2008. ISSN 1540-7977. doi: 10.1109/MPE.2008.927476.

[64] V.C. Gungor, D. Sahin, T. Kocak, S. Ergut, C. Buccella, C. Cecati, G.P. Hancke, Smart grid technologies: communication technologies and standards, IEEE Trans. Indu. Inform. 7 (4) (2011) 529-539. 